\title{
MULHERES COM CÂNCER DE MAMA EM QUIMIOTERAPIA E SUA INFLUÊNCIA SOBRE O TRABALHO*
}

\author{
Valéria Moro Maieski ${ }^{1}$, Leila Maria Mansano Sarquis ${ }^{2}$
}

\begin{abstract}
RESUMO: O estudo compreende as mudanças que o câncer de mama e a quimioterapia trouxeram para a realização das atividades laborais, em casa ou no trabalho, pelas pacientes portadoras de neoplasia mamária do ambulatório de quimioterapia de um hospital especializado em oncologia na cidade de Curitiba, em 2006. O objetivo foi compreender o cotidiano da vida da mulher com neoplasia mamária, levando em consideração a sua vivência no trabalho. O método foi o descritivo quantitativo e a população estudada foi 22 pacientes. Das mulheres que desempenhavam atividade profissional, quase a totalidade está afastada de seus trabalhos e recebem auxílio doença. A maioria refere dificuldades para a realização da atividade laboral, e impossibilidade de manter uma jornada de trabalho adequada devido a quimioterapia ou pela limitação funcional de membros superiores. Ficou caracterizada a participação de parte destas mulheres na contribuição do sustento familiar, improdutividade e limitação para o trabalho.
\end{abstract}

PALAVRAS-CHAVE: Neoplasias mamárias; Quimioterapia; Trabalho.

\section{WOMEN SUFFERING FROM BREAST CANCER UNDER CHEMOTHERAPY AND ITS INFLUENCE ON WORK PERFORMANCE}

\begin{abstract}
The study focuses on the changes that breast cancer and chemotherapy brought to task fulfillment at home or at work in patients suffering from mammary neoplasm at a chemotherapy outpatient clinic of an oncological hospital in the city of Curitiba, in 2006. It objectified to understand the daily life of women suffering from mammary neoplasm, taking into account their working experience. The method applied was descriptive-quantitative, and the population studied was 22 patients. From the working women, almost all have obtained a sick-leave and have been under the sickness aid program. The majority refers to difficulties to perform their professional activities, and the impossibility of keeping an adequate working journey due to the chemotherapy or to the functional constraint of their arms. It was evidenced that part of these women contributes for family's breadwinning, their current disability and working constraints. KEYWORDS: Breast neoplasms; Chemotherapy; Work.
\end{abstract}

\section{MUJERES COM CÁNCER DE MAMA EM QUIMIOTERAPIA Y SU INFLUENCIA SOBRE EL TRABAJO}

RESUMEN: El estudio comprende los cambios que el cáncer de mama y la quimioterapia traen para la realización de actividades laborales, sea en casa o en el trabajo, por las pacientes portadoras de neoplasia mamaria del ambulatorio de quimioterapia de un hospital especializado en oncología en la ciudad de Curitiba, 2006. Los objetivos fueron entender el cotidiano de la mujer con neoplasia mamaria, llevando en consideración su vivencia en el trabajo. El método fue el descriptivo y cuantitativo. La población estudiada fue de 22 pacientes. De las mujeres que desarrollaban actividad profesional, casi todas están lejos de su trabajo y reciben una ayuda por enfermedad. La mayoría dice haber dificultades para la realización de la actividad laboral e imposibilidad de mantener una jornada de trabajo adecuada debido a la quimioterapia o por limitación de las funciones de los miembros superiores. Se caracterizó la participación de parte de esas mujeres en la contribución del sustento familiar, improductividad y limitación para el trabajo.

PALABRAS CLAVE: Neoplasias de la mama; Quimioterapia; Trabajo.

\footnotetext{
*Artigo a partir da Monografia de conclusão de Graduação em Enfermagem da Universidade Federal do Paraná -UFPR.

${ }^{1}$ Enfermeira. Aluna do curso de especialização de Emergência em Enfermagem da Pontifícia Universidade Católica do Paraná - PUCPR.

${ }^{2}$ Enfermeira. Doutora em Enfermagem. Professora. Departamento de Enfermagem da UFPR. Membro do Grupo Estudo Multidisciplinar da Saúde do Adulto (GEMSA).
}

Autor correspondente:

Valéria Moro Maieski

R. Emílio Cornelsen, 376 - 80540-220 - Curitiba-PR.

Recebido:30/03/2007

Email: valeriammaieski@yahoo.com.br 


\section{INTRODUÇÃO}

A mama apresenta importância para o corpo da mulher como parte simbólica e característica da imagem feminina, faz relação com a sexualidade e também com a função de mulher. $O$ termo câncer de mama diz respeito à neoplasia do tecido mamário $\mathrm{e}$ também pode ser diagnosticada em homens.

Tratando-se de câncer de mama, a população feminina é a mais atingida, sendo geralmente diagnosticado mais freqüentemente nas mulheres mais velhas ou com nível de instrução maior, ou em países e regiões mais desenvolvidas, se considerando com a maioria da população feminina ${ }^{(1)}$.

$\mathrm{O}$ trabalho adquire uma importância central na vida das pessoas ${ }^{(2)}$, e para a mulher que trabalha dentro ou fora de casa, independente de seu grau de instrução, a vivência do trabalho geralmente é transformada pelo câncer. Na perspectiva de compreender o cotidiano da vida da mulher com neoplasia mamária, surgiu o interesse por esta pesquisa, a qual também leva em consideração a sua vivência do trabalho, área esta que cada vez mais tem ocupado lugar em sua vida.

Percebe-se atualmente a existência de mulheres com boa instrução, com vida razoavelmente estável, e que apesar disso ignoram informações básicas relativas à doença e o tratamento ${ }^{(3)}$. Porém, deve-se considerar que existe um conjunto de fatores que podem contribuir para que a doença se manifeste em determinadas pessoas, como o estilo de vida, dieta, predisposição familiar, consumo de álcool e comportamento reprodutivo, dentre outros, facilitando o surgimento precoce desta patologia, ou mesmo, de sua evolução sem controle.

O câncer de mama talvez represente $o$ câncer mais temido entre a população feminina, seja pelo trauma psicológico quanto à doença, o tratamento e o medo da mutilação e distorção da auto-imagem, comprometendo o aspecto físico, psicológico e social.

Este tipo de câncer ainda "é diagnosticado tardiamente em cerca de $60 \%$ dos casos" $"(4: 25)$. Somente a detecção precoce pode aumentar a perspectiva e qualidade de vida futura dessas mulheres.

A saúde comprometida pode também dificultar a presença do indivíduo em seu ambiente de trabalho. $\mathrm{O}$ afastamento do trabalho e a necessidade de se obter aposentadoria compulsória se constituem tanto do trabalho remunerado como das tarefas domésticas, em um momento de profunda crise e de estresse social para a vida da mulher. Assim, afastar-se do mercado de trabalho e do ambiente familiar para a realização do tratamento, o trauma emocional, decorrente de medo ou distorção da auto-imagem, resultante da perda da mama, pode gerar um efeito devastador para a vida da mulher. Neste sentido, o objetivo deste estudo foi identificar as conseqüências do câncer de mama e da quimioterapia na vida da mulher, enfocando as principais mudanças ocorridas durante a realização de suas atividades diárias de trabalho.

\section{REFERENCIAL TEÓRICO}

Baseada na Teoria dos Sistemas, Fernandes ${ }^{(3)}$ relata em sua obra o cotidiano da mulher com câncer de mama de forma abrangente, destacando a família e sua importância como suporte social, e a vida profissional e social da mulher diante das limitações causadas pela doença. Descreve também a transformação da vida conjugal e sexual, a autoimagem e simbolismo da mama para o corpo da mulher e seu valor cultural e, em particular suas alterações pessoais, psíquicas e sociais.

A importância do trabalho para o cotidiano do indivíduo é destacado ${ }^{(2)}$ como necessidade e fonte de realização. A juventude é vista como o momento da descoberta de aptidões em relação ao trabalho. Na fase adulta, ocorre a concretização do trabalho e o aperfeiçoamento de habilidades e, na velhice a chegada da aposentadoria e o descanso. Socialmente o indivíduo produz para o Estado e, assim, contribui para a sociedade.

\section{METODOLOGIA}

Nesta pesquisa o método utilizado foi descritivo-quantitativo. A pesquisa foi realizada em 2006, com mulheres em tratamento oncológico em local de referência, situado em Curitiba.

A amostra contou com 22 pacientes. O critério de inclusão na pesquisa baseou-se em mulheres com diagnóstico de câncer de mama, em tratamento quimioterápico adjuvante, sendo submetidas anteriormente à cirurgia de mama, tendo iniciado a quimioterapia há aproximadamente um mês, entre o ano de 2005 e 2006, com idade entre 18 e 65 anos, excluindo aquelas que apresentavam doença metastática.

O projeto foi aprovado pelo Comitê de Ética da instituição, sob o $\mathrm{n}^{\mathrm{o}}$. 1311. Os dados foram obtidos mediante entrevista com um instrumento composto por perguntas abertas e fechadas. Este, teve o objetivo de obter dados relevantes à toda atividade laboral das 
pacientes influenciada pelo câncer e o tratamento adjuvante quimioterápico.

As participantes receberam esclarecimento a respeito da pesquisa e após assinaram o Termo de Consentimento Livre e Esclarecido.

As pacientes foram selecionadas previamente em cada dia de coleta através da busca prévia e aleatória em prontuários. Algumas entrevistas foram realizadas durante o período em que estavam recebendo a quimioterapia, e outras ao término da sessão, dentro da unidade e próximo à sala de administração medicamentosa. A coleta de dados foi realizada no período de 12 de abril a 18 de maio de 2006.

Os dados foram armazenados e tabulados. Para os cálculos das freqüências relativas e absolutas, foi utilizada planilha eletrônica, dispondo os resultados em gráficos e tabelas. Os resultados foram apresentados, analisados e discutidos de acordo com a freqüência absoluta e relativa, sendo visualizados em números e porcentagens da população da pesquisa.

\section{RESULTADOS}

Houve predomínio de $32 \%$ de mulheres na faixa etária entre 31 e 40 anos. É possível que o aumento da população geral tenha causado também o crescimento da população feminina jovem, determinando, assim, o aumento no número absoluto de casos nesta faixa etária, não mudando a incidência entre mulheres ${ }^{(5)}$.

O nível de instrução concentrou-se em 36\% no ensino fundamental completo, o que é relevante ao considerarmos que no Brasil, existe grande diversidade de características socioeconômicas entre as várias regiões e, assim, a presença de diversos padrões de ocorrência da doença ${ }^{(6)}$.

Apesar da presença de histórico de câncer de mama familiar ser fator determinante para a doença ${ }^{(7)}$, neste estudo foram encontrados $80 \%$ dos casos sem correlação familiar. Esta relação prova na literatura que a incidência de casos com predisposição hereditária são esporádicas, e representam uma taxa aproximada de $10 \%{ }^{(8,9)}$.

Encontramos que 64\% das participantes faziam acompanhamento com exames complementares, mamografia e o auto-exame da mama. Os tratamentos realizados predominaram entre a cirurgia, a quimioterapia adjuvante e a radioterapia em $55 \%$ dos casos. É visto que existe a tendência de se fazer um tratamento mais eficaz dispondo de várias modalidades terapêuticas ${ }^{(10)}$.
TEMPO DE TRATAMENTO ONCOLÓGICO
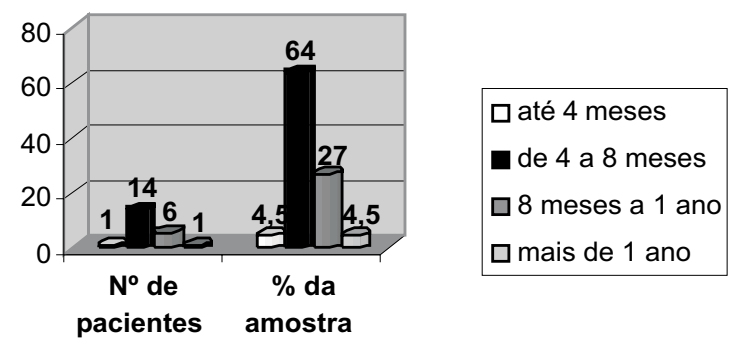

Figura 1 - Determinação da média do tempo de tratamento oncológico da população estudada. Curitiba, 2006.

O tempo médio de tratamento oncológico nesta amostra foi de 4 a 8 meses em $64 \%$. A literatura confirmou a longa permanência para este tipo de tratamento, pois se considerarmos somente a quimioterapia adjuvante, esta poderá durar em torno de 3 a 6 meses $^{(11)}$.

$\mathrm{O}$ esquema mais comum apresentado foi o FAC (fluoracil - adriamicina-ciclofosfamida) com 41\% e esquemas que contenham adriamicina, são mais efetivos que o CMF (ciclofosfamida-metrotexatefluoracil), também muito utilizados, principalmente em casos de metástase axilar ${ }^{(12)}$.

Uma porcentagem de $41 \%$ das entrevistadas realizavam o $2^{\circ}$ ou $3^{\circ}$ ciclo, em esquemas de $21 \mathrm{em} 21$ dias. Constata-se através da literatura que a mulher que passa pela quimioterapia adjuvante enfrenta, cerca de 4 meses ( 6 ciclos) a 6 meses ( 8 ciclos) de tratamento, com intervalos de 21 dias entre as aplicações, tempo suficiente para a recuperação medular ${ }^{(12)}$.

$\mathrm{O}$ tratamento cirúrgico conservador como a quadrantectomia com esvaziamento axilar foi o mais encontrado (32\%). Apesar da mastectomia ser considerada a ideal ${ }^{(13)}$, existe uma tendência atual de se fazer cirurgias parciais ou segmentadas. O problema da cirurgia mutiladora é a ocorrência de alterações psíquicas, envolvendo morbidades e a interferência na qualidade de vida na mulher, o que levou à uma mudança de conduta cirúrgica atual.

A mama esquerda é considerada a mais atingida por nódulos indolores ou espessamento de massa ${ }^{(11)}$, mas o estudo realizado não mostrou diferença estatística, com predominância de $50 \%$ da mama direita atingida, seguido de $41 \%$ da mama esquerda e de $9 \%$ de casos bilaterais. $\mathrm{O}$ auto-exame e outros métodos de diagnósticos devem ser realizados independentemente da mama.

O carcinoma ductal infiltrativo foi o diagnóstico médico mais encontrado em $86 \%$ dos casos, o que 
vem ao encontro com a literatura quando afirma ser este o tipo histológico mais freqüente ${ }^{(8,14)}$.

Neste grupo $91 \%$ das mulheres geraram filhos. Este resultado contrapõe à literatura, quando afirma que a maternidade e o maior número de filhos pode ser um fator protetor para o câncer de mama ${ }^{(15)}$. Isto envolveria também a questão da amamentação como fator de proteção ${ }^{(16)}$.

Os resultados mostram que das 20 mulheres que tiveram filhos, 15 destas geraram seus filhos antes dos 30 anos de idade. A grande maioria gerou seus filhos antes dos 30 anos e foi atingida pela doença, contrapondo também à idéia da nuliparidade e da idade tardia ao ter o primeiro filho, após os 30 anos de idade, como fatores determinantes para o desenvolvimento da doença ${ }^{(15)}$.

Quadro 1 - Tipos de profissões segundo o número de pacientes. Curitiba, 2006

\begin{tabular}{|lr|lr|}
\hline \multicolumn{4}{|c|}{ PROFISSÕES } \\
\hline Do Lar & 4 & Jardineira & 1 \\
Balconistas e Atendentes & 3 & Cobradora de ônibus & 1 \\
Vendedoras & 2 & Auxiliar de RX & 1 \\
Cozinheiras & 2 & Citotécnica & 1 \\
Garçonete e DJ & 1 & Voluntária em trabalho social & 1 \\
Artesã & 1 & Diaristas & 1 \\
Zeladora & 1 & Desempregadas & 2 \\
\hline TOTAL & \multicolumn{2}{|l}{} \\
\hline
\end{tabular}

Grande parte das profissões acima encontradas coincide com 10 profissões presentes na literatura como cargos ocupados por mulheres que trabalham em ocupações tradicionais ${ }^{(17,18)}$. A condição de gênero aparece como fator de diferença entre as ocupações de homens e mulheres, e esta divisão é chamada de "estrutura invisível derivada da divisão da sociedade em gêneros"(18:30). Entre as mulheres de classe social menos favorecida, a atividade laboral ainda é baseada no processo de divisão de função dentro da própria família ${ }^{(19)}$. Pode-se compreender então, que esta divisão é, muitas vezes, historicamente imposta.

TRABALHO OU ATIVIDADE ATUAL

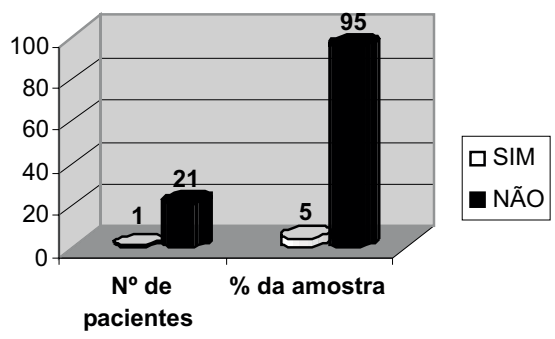

Figura 2 - Presença de atividade laboral entre mulheres com câncer de mama. Curitiba, 2006
Os resultados mostram que $95 \%$ das mulheres não desempenhavam um trabalho ou atividade fora de casa enquanto realizavam o tratamento. Esta situação vivenciada por causa da doença e do tratamento sugere que houve interferência no processo de trabalho entre estas mulheres. Socialmente a mulher durante o tratamento, é impedida de desempenhar papéis que antes eram executados por ela, o que implica afirmar que a atividade profissional está, muitas vezes, comprometida $^{(3)}$.

\section{AFASTAMENTO DO TRABALHO}

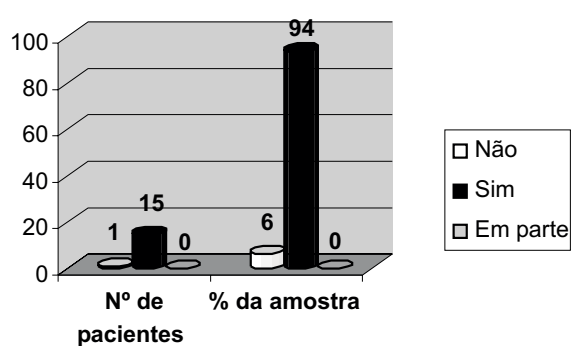

Este gráfico considera 16 mulheres em atividade profissional no momento da descoberta do diagnóstico da doença.

Figura 3 - Representação esquemática em relação ao afastamento de trabalho após o início do tratamento oncológico. Curitiba, 2006

Das 16 mulheres que trabalhavam no momento em que descobriram a doença, $94 \%$ foram afastadas totalmente de suas atividades profissionais, devido ao início do tratamento oncológico. Várias já estavam recebendo aposentadoria por auxílio doença. As demais não trabalhavam fora ou estavam desempregadas antes do início do tratamento. A mulher em seu cotidiano, pela ocorrência da doença, muitas vezes é obrigada a deixar o seu emprego e obter aposentadoria compulsória ${ }^{(3)}$.

O maior motivo do afastamento, apontado por $36 \%$ das mulheres, foi a cirurgia da mama e a quimioterapia. A dor relacionada não só ao pósoperatório, mas também de síndromes neuropáticas que podem se desenvolver, seja pela cirurgia, quimioterapia ou radioterapia. Há dificuldades para a paciente desempenhar suas funções pessoais durante a quimioterapia, gerando estresse e conflitos em sua vida pessoal e profissional, levando à um grande impacto em sua qualidade de vida ${ }^{(1)}$.

Os dados confirmam que $41 \%$ das mulheres ajudavam no complemento financeiro da família. A existência de mudanças nas relações familiares, na atualidade, diante da emancipação da mulher e sua entrada no mercado de trabalho, tornam o homem e a 


\section{RESPONSABILIDADE PELO SUSTENTO DA} FAMÍLIA

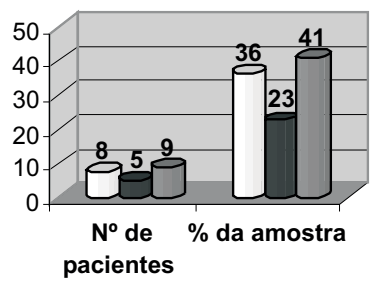

$\square N \tilde{O}$

$\square$ SIM

$\square$ EM PARTE

pacientes

Figura 4 - Presença de responsabilidade pelo sustento familiar entre mulheres com câncer de mama. Curitiba, 2006

mulher provedores materiais da família e a desenvolver papéis, às vezes, igualitários ${ }^{(19)}$.

REALIZAÇÃO DE ATIVIDADES DOMÉSTICAS

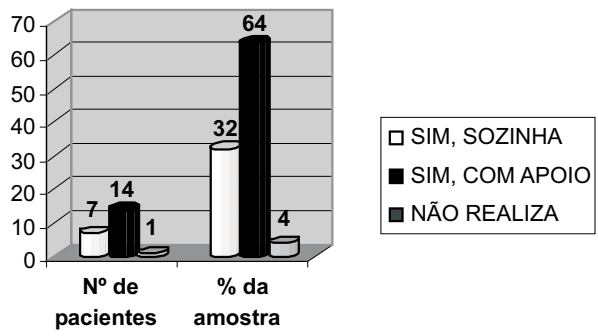

Figura 5 - Porcentagem da realização das atividades domésticas em mulheres durante a quimioterapia. Curitiba, 2006

Das 22 mulheres, 64\% ainda realizavam atividades domésticas e com a ajuda de outras pessoas, geralmente filhas, irmãs, tias ou diaristas. Ressalta-se a importância de considerar as informações sociais dessas mulheres, como a disponibilidade de recursos e de pessoas de apoio ${ }^{(20)}$. Este apoio deve ser investigado pelo enfermeiro a fim de se estabelecer relações de ajuda entre a família neste momento do tratamento, em que a diminuição das atividades e as limitações físicas são visíveis.

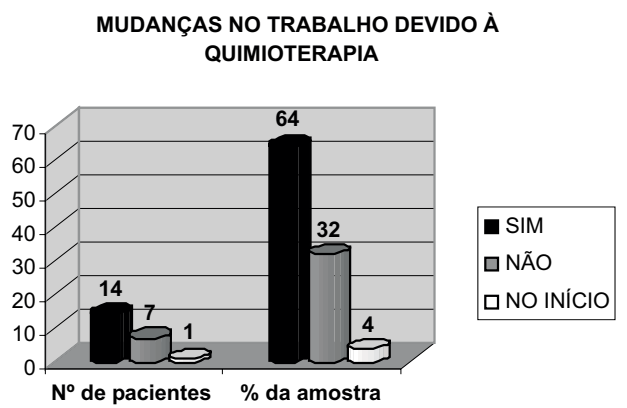

Figura 6 - Porcentagem da presença de mudanças na realização do trabalho durante a quimioterapia. Curitiba, 2006
A maioria das mulheres, $64 \%$ referiram ocorrer mudanças na realização de suas atividades devido à quimioterapia. A ausência do trabalho e da vida social é algo difícil, a sensação da interrupção da vida e a dependência da ajuda de outras pessoas ou mesmo, pela dificuldade de desempenhar sozinhas as suas atividades corriqueiras. A limitação para a maioria está presente. As que referiram ter boa tolerância ou poucas limitações representam $8 \%$ da amostra.

É possível compreender que através da realidade destas mulheres, o trabalho em casa está presente para a quase totalidade do grupo, podendo-se compreender a literatura, quando afirma que o trabalho doméstico foi por muito tempo ignorado socialmente, sendo visto como um natural prolongamento das características femininas, tanto em casa quanto no trabalho profissional, permitindo ignorar os riscos específicos que o trabalho doméstico representa para a saúde física e mental, ou delegando à mulher a maior carga de trabalho quando tem ou não um emprego ${ }^{(21)}$.

Independentemente da natureza do trabalho, percebemos que o importante para essas mulheres está no sentimento de valorização como colaboradora nas atividades, seja diante da família ou no trabalho fora de casa.

\section{COMPROMETIMENTO FUNCIONAL DE MEMBROS SUPERIORES}

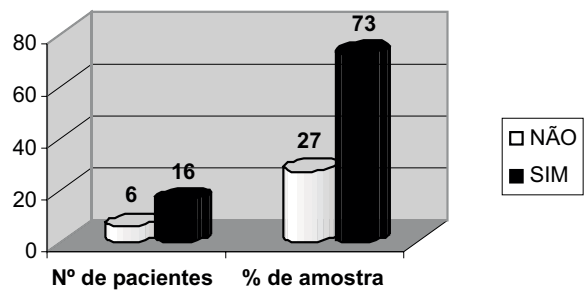

Figura 7 - Caracterização do comprometimento de membros superiores em relação ao desenvolvimento das atividades físicas. Curitiba, 2006

Para $73 \%$ das mulheres o comprometimento físico dos membros superiores causam dificuldades durante o exercício de suas atividades. As queixas são a sensação de limitação da amplitude de movimentos, não podendo elevar as mãos à cabeça ou retirar uma roupa, dificuldade em distender o braço, edema ao desenvolver os movimentos, ou mesmo em climas quentes e ao cozinhar.

Outras queixas como dor, restringe os movimentos e podem comprometer as atividades laborais, sensação de parestesia e amortecimento, 
compromete a atividade física. Os movimentos bruscos ficam comprometidos e também, pequenas atividades domésticas como varrer uma área.

A literatura confirma estes problemas quanto ao risco do edema ou mesmo de ocorrer o linfedema, quando o diâmetro do braço aumenta, causando estresse da paciente em relação à doença, aumento do peso, modificação estética e diminuição da capacidade funcional do membro acometido, os riscos de aumentar a possibilidade de infecção no braço, afetando a sua qualidade de vida ${ }^{(11,22)}$.

A existência da dor e síndromes neuropáticas, presença de ombro congelado pós-operatório, sendo necessário a reabilitação através da fisioterapia, e a síndrome da dor fantasma em mama mastectomizada, queixas que podem estar presente na vida destas pacientes $^{(23,24)}$.

A respeito dos efeitos colaterais decorrentes do tratamento quimioterápico, constatamos a presença de forma efetiva para $82 \%$ das mulheres. As reações mais presentes são vômito, enjôo, fraqueza, diarréia, alopécia, sonolência ou dificuldade para dormir, tontura, inapetência, mal estar, depressão e desânimo.

Em 68\% dos casos encontramos limitação em desempenhar as atividades decorrente dos efeitos colaterais e, entre estas mulheres, grande parte refere apresentar reações num período aproximado de 5 a 10 dias. Estas descrições confirmam sobre a maneira como a quimioterapia é vista pela maioria dos pacientes, quando relatam sobre a dificuldade que se cria em torno destes efeitos, na vivência desta situação em relação à vida profissional e social ${ }^{(1)}$.

\section{CONSIDERAÇÕES FINAIS}

A predominância do grupo estudado foi de mulheres relativamente jovens e de instrução em nível de ensino fundamental, considerando que a característica do atendimento de pacientes da instituição pesquisada, em sua maior parte, é representada através do SUS, e o atendimento por convênios particulares é em torno de $14 \%$.

Levando em consideração que a mulher desempenha também como responsabilidade o sustento e o complemento de recursos materiais na sua família, podemos constatar que a doença oncológica pode comprometer socialmente e psicologicamente a vida destas mulheres. Grande parte das mulheres entrevistadas ajuda no sustento de suas famílias.

O estudo atingiu o objetivo quando comprovou que $73 \%$ das mulheres entrevistadas trabalhavam fora de casa no momento em que descobriram a sua doença, e se afastaram por motivo da doença. Percebemos, a dimensão que o câncer de mama acarreta para a sociedade frente a questão social e econômica. $\mathrm{O}$ afastamento do mercado de trabalho destas mulheres, muitas vezes definitivo, representa não só a perda e o prejuízo pessoal, mas também, perdas de pessoas economicamente ativas, que podem não retornar ao mercado de trabalho, sendo necessário o provimento de aposentadorias antecipadas por auxílio doença. É necessário considerar ainda, além do aspecto da ocupação e atividade, a dimensão de todo o prejuízo emocional, físico e mental que acarreta na vida da mulher, seja na figura e no desempenho de seu papel como mulher, mãe, esposa e também trabalhadora. A atribuição de suas funções talvez possa transformar-se, modificando a estrutura de sua vida de modo permanente.

Por isso, a mulher que passa pelo tratamento do câncer de mama deve ser reabilitada em seus vários aspectos, físico, emocional, social e profissional. A superação de certas dificuldades pode ser minimizada pelos profissionais enfermeiros e demais equipe, que percebam e compreendam o seu drama e sejam conhecedores desta causa, já que o câncer de mama é um agravo de incidência significativa na saúde de nosso país e, também em nível mundial, por isso se torna tão necessária a prevenção.

Devemos reavaliar o papel do enfermeiro e da medicina em saúde coletiva, diante da questão da detecção e prevenção do câncer de mama, como a mulher tem sido ouvida e avaliada em suas queixas habituais em relação à sua saúde, e como os profissionais estão realizando a sua prática. Outra preocupação é de que forma podemos atuar para minimizar tais agressões que o tratamento oncológico impõe.

As causas reais do câncer de mama ainda são estudadas por muitos profissionais de saúde, o que nos leva a pensar sobre o seu aspecto multifatorial, e o quanto compete à Enfermagem o empenho e aprimoramento nesta questão, visando também à educação e alcance da população feminina, a mais atingida pelo problema.

\section{REFERÊNCIAS}

1 Bassegio DL, Soldatelli MM. Aspectos psicossociais no câncer de mama. In: Baseglio DL. Câncer de mama: abordagem multidisciplinar. Rio de Janeiro: Revinter; 1999. p.353-5.

2 Souto DF. Saúde no trabalho: uma evolução em 
andamento. Rio de Janeiro: Senac Nacional; 2003.

3 Fernandes AFC. O cotidiano da mulher com câncer de mama. Fortaleza: Fundação Cearense de Pesquisa e Cultura; 1997.

4 Ministério da Saúde (BR). Breve diagnóstico da situação da saúde da mulher no Brasil. Política Nacional de Atenção Integral à Saúde da Mulher - Princípios e Diretrizes. Distrito Federal: MS; 2004.

5 Nsajon LW, Balem JL. A evolução do câncer de mama na mulher jovem - uma revisão de literatura. Instituto de Ginecologia-UFRJ. Revista Feminina. 1999;27(9):745-7.

6 Frasson A, Koch H, Freud L. Manejo das lesões mamárias não-palpáveis. In: Basegio DL. Câncer de mama: abordagem multidisciplinar. Janeiro: Revinter; 1999.p. 123.

7 Menke CH, Biazús JV, Cavalheiro JA, Rabin EG. Diagnóstico clínico do câncer de mama. In: Basegio DL. Câncer de mama: abordagem multidisciplinar. Rio de Janeiro: Revinter; 1999. p.17-24.

8 Norton L; Gilewiski T. Câncer de mama. In: Kelley WN. Tratado de medicina interna. $3^{\mathrm{a}}$ ed. Rio de Janeiro: Guanabara Koogan; 1999.

9 Basegio DL, Webber L, Hamerski MG. Manejo da paciente de alto risco. In: Basegio DL. Câncer de mama: abordagem multidisciplinar. Rio de Janeiro: Revinter. 1999.p.145-9.

10 Colman FT; Salvajoli JV. Ação potencializadora da quimioterapia sobre a radioterapia. In: Ayoub AC, Frias MAE, Barros MA, Kobaiashi RM. Bases da enfermagem em quimioterapia. São Paulo: Lemar; 2000. p.146-7.

11 Entrekin N. Cãncer de mama. In: Clark JC, Mc Gee, RF. Enfermagem oncológica: um curriculum básico. $2^{\mathrm{a}} \mathrm{ed}$. Porto Alegre: Artes Médicas; 1997. p.401-15.

12 Teixeira LC. Quimioterapia adjuvante no câncer de mama. In: Baseglio DL. Câncer de mama: abordagem multidisciplinar. Rio de Janeiro: Revinter; 1999. p.223.

13 Brenelli HB; Kepkke EM. Reconstrução mamária. In: Basegio DL. Câncer de mama: abordagem multidisciplinar. Rio de Janeiro: Revinter; 1999. p.210-2.

14 Basegio DL; Lersch E. Classificação histopatológica do câncer de mama. In: Basegio DL. Câncer de mama: abordagem multidisciplinar. Rio de Janeiro: Revinter; 1999. p.12-6.

15 Tessaro S. Epidemiologia do câncer de mama-cap. 1.
In: Basegio DL. Câncer de mama: abordagem multidisciplinar. Rio de Janeiro: Revinter; 1999. p.1-9.

16 Rea MF. Os benefícios da amamentação para a saúde da mulher. J Ped. 2004;80(5 Supl):142-6.

17 Stellman JM. Mulheres no trabalho. In: Bowler RM, Cone JE. Segredos em medicina do trabalho. São Paulo: Artmed;2001.p.215-9.

18 Andrade MLAG. A saúde no trabalho de homens e mulheres: uma visão de gênero. In: Oliveira EM; Scavone L. Trabalho, saúde e gênero na era da globalização. Goiânia: AB; 1997. p.29-32.

19 Sarti CAV. Os filhos dos trabalhadores: quem cuida das crianças? In: Oliveira EM; Scavone L. Trabalho, saúde e gênero na era da globalização. Goiânia: AB; 1997. p.51-9.

20 Smeltzer SC; Bare, BG. Histórico e tratamento de pacientes com distúrbios da mama. In: Brunner/ Suddarth - Tratado de enfermagem médico-cirúrgica. $8^{a}$ ed. Rio de Janeiro: Guanabara Koogan; 2002. p.120238 .

21 Romito P. Trabalho, maternidade e saúde: algumas notas metodológicas. In: Oliveira EM; Scavone L. Trabalho, saúde e gênero na era da globalização. Goiânia: AB; 1997.p. 15-27.

22. Freitas Junior R; Ribeiro LFJ; Kajita D. Linfedema pósmastectomia. In: Basegio DL. Câncer de mama: abordagem multidisciplinar. Rio de Janeiro: Revinter; 1999.p. 346

23. Bittencourt RKR; Basegio DL. Tratamento da dor no Câncer de mama. In: Basegio DL. Câncer de mama: abordagem multidisciplinar. Rio de Janeiro: Revinter. 1999.p. 333-43

24. Safiano CM, Sarquis LMM, Felli VEA, Giacomozzi LM. O processo saúde doença vivenciado pelos trabalhadores de enfermagem em uma instituição hospitalar. Cogitare Enferm. 2003 Jul/Dez;8(2):87-91

Cogitare Enferm 2007 Jul/Set; 12(3):346-52 